\title{
Tailles de première maturité et de capture de six espèces de poisson-chat dans le delta de l'Ouémé au Bénin (Afrique de l'Ouest)
}

\author{
Antoine CHIKOU ${ }^{1 *}$, Philippe A. LALEYE ${ }^{1}$, Clément A. BONOU ${ }^{2}$, \\ Pierre VANDEWALLE ${ }^{3}$ et Jean-Claude PHILIPPART ${ }^{4}$ \\ ${ }^{1}$ Université d'Abomey-Calavi, Faculté des Sciences Agronomiques, Laboratoire d'Hydrobiologie et \\ d'Aquaculture, 01 BP 526 Cotonou, Bénin. Tél : (229)21046406 ou (229)90023036) [chikoua@yahoo.fr et \\ laleyeph@yahoo.fr]. \\ ${ }^{2}$ Université d'Abomey-Calavi, Ecole Polytechnique d'Abomey-Calavi, Laboratoire d'Hydrobiologie Appliquée, \\ 01 BP 2009 Cotonou, Bénin. Tél : (229)90925123 [acbonou@yahoo.fr]. \\ ${ }^{3}$ Université de Liège, Laboratoire de Morphologie Fonctionnelle et Evolutive, Institut de Chimie, B 6 Sart \\ Tilman, B 4000 Liège, Belgique. Tél : (32)43665040 ou(32)43665024 [P.Vandewalle@ulg.ac.be]. \\ ${ }^{4}$ Université de Liège, Laboratoire de Démographie des Poissons et d'Hydroécologie, 10 Chemin de la justice, \\ B 4500 Tihange, Belgique.Tél : (32)19328301 ou (32)85274155 [jcphilippart@ulg.ac.be]. \\ *Auteur correspondant, E-mail : chikoua@yahoo.fr; Tél : (229)90023036 ou 96691353.
}

\section{RESUME}

Dans le moyen delta de l'Ouémé au Bénin, les tailles de première maturité et de capture des populations de six espèces de poisson-chat ont été étudiées. Il s'agit de Clarias gariepinus, de C. ebriensis, de Schilbe intermedius, de $S$. mystus, de Synodontis schall et de $S$. nigrita. La relation de type sigmoïde entre le pourcentage de maturité sexuelle $(\mathrm{P})$ et la taille $(\mathrm{Lt})$ des poissons a été déterminée d'après le modèle logistique de Dagnelie (1973). L'équation générale de Von Bertalanffy (1938) incorporée au logiciel FISAT II a permis de déterminer les tailles de première capture $\left(\mathrm{Lc}_{50}\right)$. En général, les mâles maturent à une taille inférieure à celle des femelles. Les tailles à la première maturité $\left(\mathrm{L}_{50}\right)$ sont supérieures aux tailles de première capture $\left(\mathrm{Lc}_{50}\right)$. On en déduit une surexploitation des six populations de poissons étudiées qui sont en général pêchées avant la première reproduction. Il serait intéressant que les poissons soient capturés à une plus grande taille $\left(\mathrm{Lc}_{50}>\mathrm{L}_{50}\right)$.

(C) 2011 International Formulae Group. All rights reserved.

Mots clés : Poissons-chats, maturité sexuelle, exploitation, delta de l'Ouémé.

\section{INTRODUCTION}

Le fleuve Ouémé, au Bénin, recèle d'importantes quantités de poissons-chats dont l'intérêt économique est de plus en plus connu, notamment dans sa partie deltaïque (Lalèyè et al., 2004, 2007 ; Chikou et al., 2007; Imorou, 2007). Ces poissons sont très appréciés dans l'alimentation humaine et la demande sur les marchés locaux et internationaux (Nigeria, Togo, Burkina Faso) est de plus en plus élevée, soumettant leurs populations naturelles à une très forte pression de pêche (Chikou, 2006). L'étude bioécologique approfondie de ce groupe de poissons dans le bassin de l'Ouémé est encore à ses débuts (Lalèyè, 1995; Lalèyè et al., 
2004, 2005, 2006, 2007 ; Chikou, 2006, 2008 ; Imorou et al., 2007). Des études pour la mise en évidence de leurs caractéristiques biologiques et démographiques, en rapport avec leur sensibilité aux facteurs environnementaux et anthropiques, s'avèrent nécessaires. Au nombre de ces études, la connaissance de la taille à la première maturité chez les poissons occupe une place de choix en vue de déterminer la taille minimale de capture et réglementer les mailles des filets de pêche (Chikou, 2006). Cette approche semble nécessaire pour la définition et la mise en œuvre d'une politique de gestion rationnelle de ces ressources. Il est donc important de laisser les poissons se reproduire une fois au moins avant de les éliminer du milieu (Lalèyè et al., 1995). L'objectif de ce travail est d'améliorer la connaissance scientifique de l'écologie et de l'exploitation halieutique des populations de Clarias gariepinus, de C. ebriensis, de Schilbe intermedius, de S. mystus, de Synodontis schall et de $S$. nigrita. Il s'agit plus spécifiquement de déterminer les tailles de première maturité et de première capture afin de proposer des stratégies de conservation de ces espèces.

\section{MATERIEL ET METHODES}

\section{Milieu d'étude}

Les données sont collectées à Agonlin Lowé (N 06³9'378", E 002²8'571"), un village situé dans le delta du fleuve Ouémé, Commune d'Adjohoun au Bénin (Figure 1). Le climat est du type subéquatorial caractérisé par quatre saisons : deux saisons des pluies d'inégale importance dont la plus grande s'étale de mars-avril à juillet et la plus petite de septembre à novembre, et deux saisons sèches dont la plus grande va de décembre à mars et la plus petite de juilletaoût à début septembre (Adam et Boko, 1993). L'humidité relative est élevée (77 à 93 $\%$ ) et la température de l'air, plus ou moins constante, est en moyenne de $27,5{ }^{\circ} \mathrm{C}$ toute l'année. Le delta de l'Ouémé connaît une seule saison de hautes eaux dans l'année et l'amplitude est très forte entre la crue (fin juillet à début novembre) et l'étiage (décembre à juin). Les activités de pêche sont intenses en période de crue dans les plaines inondées mais se poursuivent toute l'année dans le lit du fleuve (Welcomme, 2002; Chikou et al., 2007; Imorou et al., 2007 ; Lalèyè et al., 2007).

\section{Collecte des données}

Les données sont collectées mensuellement de mai 1999 à avril 2000 et tous les deux mois de mai 2000 à mars 2001. Les pêches ont eu lieu dans le lit du fleuve, dans les Acadjas qui sont des parcs à poissons (Welcomme, 2002 ; Lalèyè et al., 2007), dans les Whédos qui sont des trous à poissons (Lalèyè et al., 2007 ; Imorou et al., 2007) et dans les plaines inondées. Neuf (9) filets maillants expérimentaux de mailles $10,12,15$, $20,25,30,35,40$ et $45 \mathrm{~mm}$ et des épuisettes à mailles fines $(1 \mathrm{~mm})$ sont utilisés. Les captures sont complétées par celles des engins de pêche artisanaux utilisés par les pêcheurs pour les pêches commerciales. Ces diverses techniques de pêche, utilisées dans différents types d'habitats, ont permis la prise en compte des poissons de toutes les classes de tailles. Les échantillons sont examinés frais par espèce. Pour chaque individu, la longueur totale ( $\mathrm{Lt}$ en $\mathrm{cm}$ ) et le poids total (Pt en $\mathrm{g}$ ) sont mesurés. Les poissons sont disséqués. Le sexe et le stade de maturité sexuelle sont déterminés par l'examen des ovaires ou des testicules du poisson sur la base de l'échelle de maturité selon Lalèyè (1995) inspirée de Micha (1973).

\section{Traitement des données et analyses statistiques}

Les données sont encodées sur un ordinateur PC et les possibilités macro du logiciel Excel 2000 ont permis de réaliser les tableaux de synthèse qui s'imposent.

Pour la détermination des pourcentages de maturité sexuelle, les femelles aux stades II à V de maturité ayant des gonades «actives » sont prises en compte ainsi que les mâles aux mêmes stades de 


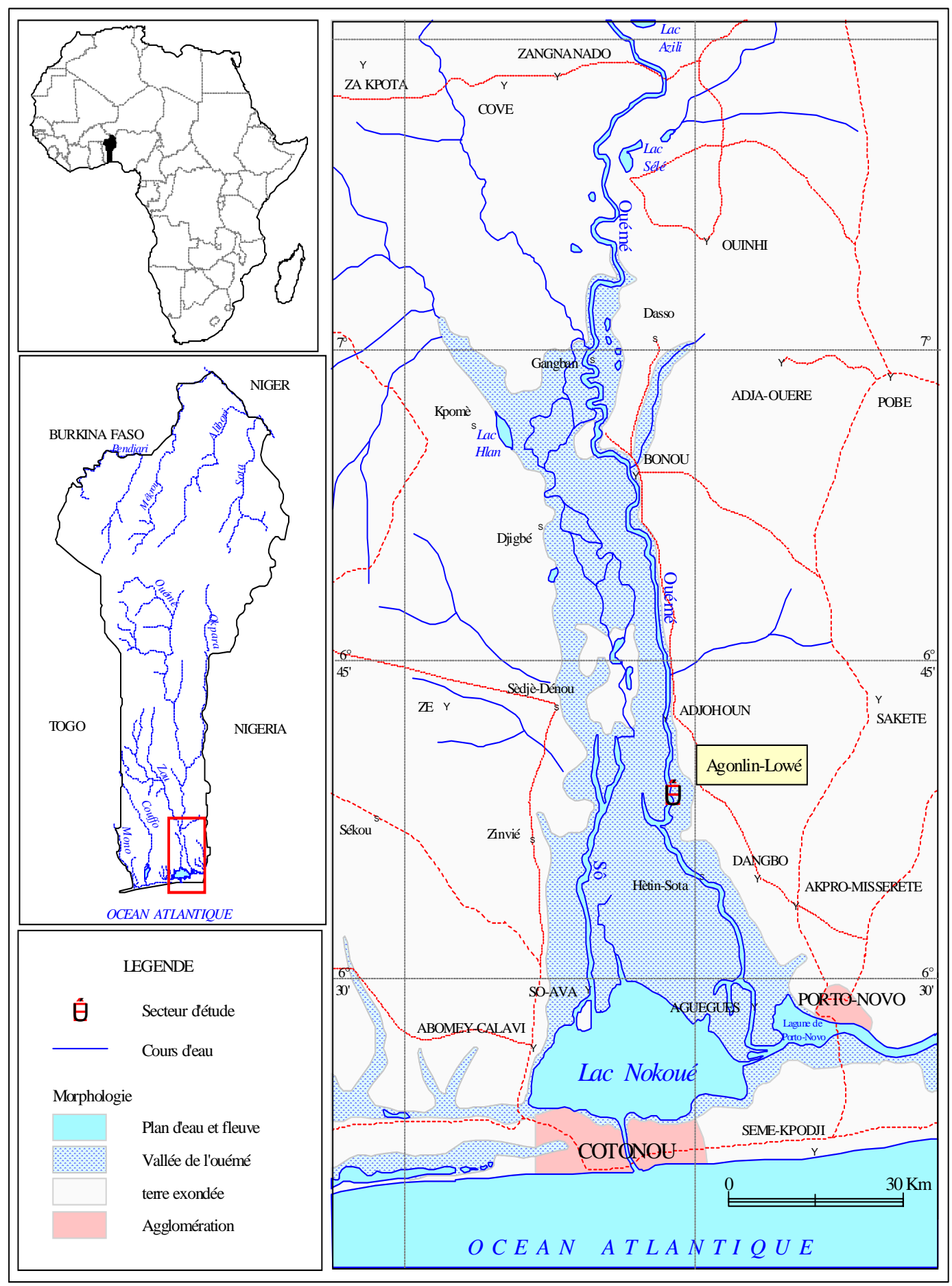

Figure 1 : Localisation de la zone d'étude. 
maturité sexuelle (Dunn, 1972; Gwahaba, 1978; Moreau, 1979). Selon Tweddle et Turner (1977), la taille de première maturité $\left(\mathrm{L}_{50}\right)$ est celle atteinte par $50 \%$ des individus des deux sexes matures pour la première fois. En général, la relation entre le pourcentage de maturité estimé (P) et la taille ( $\mathrm{Lt}$ ) est de type sigmoïde. L'équation de la courbe sigmoïde déterminée d'après le modèle logistique est donnée selon Dagnelie (1973) par la formule :

$$
\begin{array}{cl}
P=X /(1+X) & \text { avec } \\
X=e^{(a+b L t)} & \text { a et b étant des }
\end{array}
$$

paramètres du modèle. La transformation logarithmique de l'équation a permis de la mettre sous la forme : $\ln \left(\frac{P}{(1-P)}\right)=a+b L t$ et, en remplaçant $P=50 \%$ dans l'équation, la $\mathrm{L}_{50}$ est obtenue par : $L_{50}=-a / b$. Ce modèle logistique de la taille de première maturité est déterminé au moyen du logiciel StatView (version 5.0.1) du Laboratoire de Démographie des Poissons et d'Hydroécologie de l'Université de Liège (Belgique).

La taille de première capture $\left(\mathrm{Lc}_{50}\right)$ est la longueur totale à laquelle $50 \%$ des poissons sont capturés par la pêche. L'équation générale de Von Bertalanffy (1938) incorporée au logiciel FISAT II (Niyonkuru, 2007) a permis de la déterminer.

Le test de Chi carré $\left(\chi^{2}\right)$ a permis de tester les différentes proportions calculées ainsi que les sex-ratios. L'analyse de la variance à un critère de classification (Anova 1) est utilisée pour comparer les variables « tailles » et « poids » au sein des populations. La signification des résultats est donnée au seuil de $5 \%$.

\section{RÉSULTATS}

\section{Caractéristiques générales des poissons}

L'étude a porté sur un effectif total de 11.445 poissons répartis par espèce et par sexe comme indiqué dans le Tableau 1. On remarque le nombre d'individus relativement faible récolté pour l'espèce $S$. mystus par rapport aux autres espèces. Ceci est lié aux exigences écologiques de cette espèce qui préfère les milieux bien oxygénés. Elle n'a été capturée en majorité que dans le lit principal du fleuve.

L'analyse des tailles (Lt) des poissons capturés (Tableau 1) indique une différence significative $(\mathrm{p}<0,05)$ entre les individus mâles et femelles chez Clarias ebriensis, Schilbe intermedius et Synodontis schall, ce qui n'est pas le cas pour les trois autres espèces (Clarias gariepinus, Schilbe mystus, et Synodontis nigrita).

\section{Sex-ratio}

Le Tableau 2 présente les sex-ratios observés par espèce dans les captures. Il apparaît que les femelles sont significativement plus nombreuses que les mâles pour $C$. gariepinus, C. ebriensis et $S$. nigrita $(\mathrm{p}<0,05)$. Ce sont les mâles qui sont significativement plus nombreux chez $S$. intermedius et $S$. schall $(\mathrm{p}<0,05)$. Pour $S$. mystus, le sex-ratio n'est pas significativement différent du sex-ratio théorique 1:1 ( $p>0,05)$.

\section{Tailles de première maturité}

Les paramètres des équations calculés du modèle logistique pour la relation «pourcentage de maturité-longueur totale du poisson» chez les espèces sont présentés dans le Tableau 3. Les graphiques de la Figure 2 donnent le pourcentage de maturité sexuelle en fonction de la longueur totale du corps (Lt) chez les six espèces étudiées ainsi que les courbes d'ajustement selon le modèle.

Chez C. gariepinus, la taille minimale des individus matures est de $13 \mathrm{~cm}$ pour les femelles et de $11 \mathrm{~cm}$ pour les mâles. A partir de $25 \mathrm{~cm}$ pour les mâles et de $33 \mathrm{~cm}$ pour les femelles, les poissons observés sont tous matures. La taille de première maturité $\mathrm{L}_{50}$ est significativement différente $(\mathrm{p}<0,05)$ pour les deux sexes $(18,1 \mathrm{~cm}$ pour les femelles et $16,2 \mathrm{~cm}$ pour les mâles). 
Tableau 1 : Tailles moyennes ( $\mathrm{Lt}$ en $\mathrm{cm}$ ), maximales, minimales et nombre total de poissons échantillonnés à Agonlin Lowé de mai 1999 à mars 2001.

\begin{tabular}{|c|c|c|c|c|c|}
\hline Espèces & Paramètres & Femelle & Mâle & Indéterminé & Total \\
\hline \multirow[b]{2}{*}{ Clarias gariepinus } & $\begin{array}{l}\text { Moyenne Lt } \\
\text { (cm) }\end{array}$ & $24,4 \pm 7,1^{\mathrm{a}}$ & $24,3 \pm 8,9^{\mathrm{a}}$ & $5,5 \pm 2,6^{\mathbf{b}}$ & $22,7 \pm 9,3$ \\
\hline & $\begin{array}{l}\text { Nbre (max ; } \\
\text { min) }\end{array}$ & $\begin{array}{c}591(63,0, \\
11,0) \\
\end{array}$ & $\begin{array}{c}75(64,0 ; \\
11,0) \\
\end{array}$ & $101(11,0 ; 1,6)$ & $\begin{array}{c}1167(64,0 ; \\
1,6) \\
\end{array}$ \\
\hline \multirow[b]{2}{*}{ Clarias ebriensis } & $\begin{array}{c}\text { Moyenne Lt } \\
(\mathrm{cm})\end{array}$ & $14,4 \pm 4,1^{\mathrm{a}}$ & $15,7 \pm 4,7^{\mathbf{b}}$ & $8,0 \pm 3,7^{\mathrm{c}}$ & $14,7 \pm 4,6$ \\
\hline & $\begin{array}{l}\text { Nbre (max ; } \\
\text { min) }\end{array}$ & $\begin{array}{c}478(26,1 ; \\
7,0)\end{array}$ & $\begin{array}{c}395(33,0 ; \\
7,2)\end{array}$ & $38(15,0 ; 2,2)$ & $\begin{array}{c}911(33,0 ; \\
2,2)\end{array}$ \\
\hline \multirow[b]{2}{*}{ Schilbe intermedius } & $\begin{array}{l}\text { Moyenne Lt } \\
(\mathrm{cm})\end{array}$ & $10,6 \pm 2,2^{\mathrm{a}}$ & $10,5 \pm 1,6^{\mathbf{b}}$ & $8,8 \pm 1,1^{\mathbf{c}}$ & $10,1 \pm 1,9$ \\
\hline & $\begin{array}{l}\text { Nbre (max ; } \\
\text { min) }\end{array}$ & $\begin{array}{c}1015(20,6 ; \\
7,3) \\
\end{array}$ & $\begin{array}{c}\mathbf{1 1 3 0} \\
(19,3 ; 7,3)\end{array}$ & $\mathbf{8 2 2}(12,7 ; 5,7)$ & $\begin{array}{c}2967(20,6 ; \\
5,7) \\
\end{array}$ \\
\hline \multirow[b]{2}{*}{ Schilbe mystus } & $\begin{array}{l}\text { Moyenne Lt } \\
(\mathrm{cm})\end{array}$ & $12,2 \pm 2,8 \mathbf{a}$ & $9,9 \pm 1,2 \mathbf{a}$ & $11,8 \pm 1,6 \mathbf{b}$ & $10,7 \pm 2,0$ \\
\hline & $\begin{array}{l}\text { Nbre (max ; } \\
\text { min) }\end{array}$ & $\begin{array}{c}64(20,8 ; \\
6,5)\end{array}$ & $\begin{array}{c}79(17,0 ; \\
9,6)\end{array}$ & $227(12,6 ; 6,5)$ & $\begin{array}{c}370(20,8 ; \\
6,5)\end{array}$ \\
\hline \multirow[b]{2}{*}{ Synodontis schall } & $\begin{array}{l}\text { Moyenne Lt } \\
(\mathrm{cm})\end{array}$ & $11,3 \pm 3,8 \mathbf{a}$ & $\begin{array}{c}10,3 \pm 3,7 \\
\mathbf{b}\end{array}$ & $7,5 \pm 1,5 \mathrm{c}$ & $9,4 \pm 3,4$ \\
\hline & $\begin{array}{l}\text { Nbre (max } \\
\text { min) }\end{array}$ & $\begin{array}{c}1197(26,2 ; \\
5,1)\end{array}$ & $\begin{array}{c}\mathbf{1 6 1 5} \\
(32,1 ; 3,8)\end{array}$ & $2041(18,5 ; 3,0)$ & $\begin{array}{c}\mathbf{4 8 5 3}(32,1 ; \\
3,0)\end{array}$ \\
\hline \multirow[b]{2}{*}{ Synodontis nigrita } & $\begin{array}{l}\text { Moyenne Lt } \\
(\mathrm{cm})\end{array}$ & $12,7 \pm 5,4 \mathbf{a}$ & $\begin{array}{c}13,1 \pm 4,1 \\
\mathbf{a}\end{array}$ & $6,9 \pm 1,9 \mathbf{b}$ & $10,6 \pm 4,9$ \\
\hline & $\begin{array}{l}\text { Nbre (max ; } \\
\text { min) }\end{array}$ & $\begin{array}{c}419(27,6 ; \\
3,8) \\
\end{array}$ & $\begin{array}{c}319(22,4 ; \\
5,4)\end{array}$ & $439(17,2 ; 3,4)$ & $\begin{array}{c}1177(27,6 ; \\
3,4) \\
\end{array}$ \\
\hline
\end{tabular}

Tableau 2: Sex-ratios calculés en fonction des espèces étudiées.

\begin{tabular}{lcccccc}
\hline Espèce & $\begin{array}{c}\boldsymbol{C} . \\
\text { gariepinus }\end{array}$ & $\begin{array}{c}\boldsymbol{C} \text {. } \\
\text { ebriensis }\end{array}$ & $\begin{array}{c}\text { S. } \\
\text { intermedius }\end{array}$ & $\begin{array}{c}\boldsymbol{S} . \\
\text { mystus }\end{array}$ & $\begin{array}{c}\text { S. } \\
\text { schall }\end{array}$ & $\begin{array}{c}\boldsymbol{S} . \\
\text { nigrita }\end{array}$ \\
\hline Sex-ratio $(\mathrm{F}: \mathrm{M})$ & $1: 0,8$ & $1: 0,83$ & $1: 1,1$ & $1: 1,2$ & $1: 1,4$ & $1: 0,8$ \\
Chi carré $\left(\chi^{2}\right)$ & $12,62^{*}$ & $7,89^{*}$ & $6,17 *$ & 1,57 & $62,14^{*}$ & $13,55^{*}$ \\
\hline$*$ sexe ratio significativement différent $(p<0,05)$ du sexe ratio théorique de $1: 1$. & & &
\end{tabular}

Chez C. ebriensis, la taille minimale des individus matures est de $6 \mathrm{~cm}$. A partir de $16 \mathrm{~cm}$ pour les mâles et de $18 \mathrm{~cm}$ pour les femelles, les poissons observés sont tous matures. On observe aussi que la taille de première maturité $\mathrm{L}_{50}$ est significativement différente $(\mathrm{p}<0,05)$ pour les deux sexes $(11,3$ $\mathrm{cm}$ pour les femelles et $7,4 \mathrm{~cm}$ pour les mâles).

A $8 \mathrm{~cm}, 44,2 \%$ des femelles et $50,8 \%$ des mâles sont matures chez $S$. intermedius. En dessous de $8 \mathrm{~cm}$, le sexe des individus capturés n'a pu être déterminé. A partir de 16 $\mathrm{cm}$ pour les mâles et de $20 \mathrm{~cm}$ pour les femelles, les poissons observés sont tous matures. La $\mathrm{L}_{50}$ est de $9,7 \mathrm{~cm}$ pour les femelles et de $8,4 \mathrm{~cm}$ pour les mâles, marquant une différence significative entre les deux sexes $(\mathrm{p}<0,05)$.

Chez S. mystus, à $12 \mathrm{~cm} \mathrm{Lt,} \mathrm{17,6 \%}$ des femelles et $11,5 \%$ des mâles sont matures chez $S$. mystus. A partir de $18 \mathrm{~cm}$ pour les mâles et de $16 \mathrm{~cm}$ pour les femelles, les poissons observés sont tous matures. $\mathrm{La}_{50}$ est de $13,6 \mathrm{~cm}$ pour les femelles et de $14,7 \mathrm{~cm}$ pour les mâles, la différence étant significative $(\mathrm{p}<0,05)$. 
La taille minimale des individus matures est de $6 \mathrm{~cm}$ chez les femelles et de 8 cm chez les mâles de $S$. schall. A partir de 20 $\mathrm{cm}$ chez les mâles et de $24 \mathrm{~cm}$ chez les femelles, les poissons observés sont tous matures. La taille de première maturité $\mathrm{L}_{50}$ est significativement différente $(\mathrm{p}<0,05)$ pour les deux sexes $(13,2 \mathrm{~cm}$ pour les femelles et $12,2 \mathrm{~cm}$ pour les mâles).

Chez $S$. nigrita, les individus matures ont une taille minimale de $8 \mathrm{~cm}$ pour les deux sexes. A partir de $22 \mathrm{~cm}$ chez les mâles et de $24 \mathrm{~cm}$ chez les femelles, les poissons observés sont tous matures. Aucune différence significative $(p>0,05)$ n'est observée entre les $\mathrm{L}_{50}$ des deux sexes $(13,1 \mathrm{~cm}$ pour les femelles et $13,0 \mathrm{~cm}$ pour les mâles).

\section{Taille de première capture}

L'analyse des probabilités de capture issues des parties gauches ascendantes de courbes de capture pour les six espèces a été réalisée (Tableau 4). Elle indique pour $C$. gariepinus que $75 \%$ des poissons sont capturés à une taille de $11,5 \mathrm{~cm}$, tandis que $100 \%$ le sont à une taille de $23,6 \mathrm{~cm}$. La taille de première capture $\mathrm{Lc}_{50}$ étant de $1,8 \mathrm{~cm}$. Pour C. ebriensis, les $75 \%$ sont capturés à $9,0 \mathrm{~cm}$ et les $100 \%$ à $18,2 \mathrm{~cm}$, pour une $\mathrm{Lc}_{50}$ de 4,82 $\mathrm{cm}$. Chez $S$. intermedius, $75 \%$ des individus sont capturés à $7 \mathrm{~cm}$ et la totalité $(100 \%)$ l'est à $9,7 \mathrm{~cm}$; la $\operatorname{Lc}_{50}$ est de $5,5 \mathrm{~cm}$, tandis que chez S. mystus $75 \%$ des individus sont capturés à $6,7 \mathrm{~cm}$ et les $100 \%$ à $8,0 \mathrm{~cm}$, pour une $\mathrm{Lc}_{50}$ de $5,5 \mathrm{~cm}$. Pour $S$. schall et $S$. nigrita, $75 \%$ des individus sont capturés respectivement à $4,0 \mathrm{~cm}$ et $5,0 \mathrm{~cm}$ tandis que la totalité $(100 \%)$ de ceux-ci l'est respectivement à 5,0 cm et $19,4 \mathrm{~cm}$. Les $\mathrm{Lc}_{50}$ sont respectivement $2,4 \mathrm{~cm}$ et $3,2 \mathrm{~cm}$.

Les longueurs maximales observées dans le milieu sont toutes proches des Lt infinies (Lto) estimées par le modèle de Von Bertalanffy, mais inférieures à celles-ci. Ce qui montre que les six espèces sont toutes capturées avant d'atteindre la $\mathrm{L} \infty$.

Tableau 3 : Paramètres du modèle logistique de la relation entre le pourcentage de maturité sexuelle $(\%)$ et la longueur totale $(\mathrm{cm})$ chez les six espèces de poisson-chat étudiées.

\begin{tabular}{|c|c|c|c|c|c|c|c|c|}
\hline \multicolumn{2}{|c|}{ Paramètres de la relation } & $\mathbf{N}$ & $\mathbf{a}$ & b & $\mathbf{r}^{2}$ & $\begin{array}{c}\mathbf{L}_{50} \\
(\mathbf{c m})\end{array}$ & $\begin{array}{l}\mathrm{L}_{100} \\
(\mathrm{~cm})\end{array}$ & $\begin{array}{c}\mathbf{L}_{50} / \mathrm{Lt} \text { max } \\
(\%)\end{array}$ \\
\hline \multirow[t]{3}{*}{ C. gariepinus } & mâles & 475 & $-7,116$ & 0,438 & 0,94 & $16,2^{\mathrm{a}}$ & $25,0^{a}$ & 25 \\
\hline & femelles & 591 & $-6,796$ & 0,376 & 0,94 & $18,1^{\text {b }}$ & $33,0^{\mathbf{b}}$ & 29 \\
\hline & ind. & 101 & & & & & & \\
\hline \multirow{3}{*}{ C. ebriensis } & mâles & 395 & $-3,701$ & 0,497 & 0,92 & $7,4^{\mathrm{a}}$ & $16,0^{\mathrm{a}}$ & 22 \\
\hline & femelles & 478 & $-4,897$ & 0,430 & 0,86 & $11,3^{\mathbf{b}}$ & $18,0^{b}$ & 43 \\
\hline & ind.. & 38 & & & & & & \\
\hline \multirow[t]{3}{*}{ S. intermedius } & mâles & 1130 & $-3,701$ & 0,439 & 0,86 & $8,4^{\mathrm{a}}$ & $16,0^{\mathrm{a}}$ & 44 \\
\hline & femelles & 1015 & $-3,343$ & 0,343 & 0,79 & $9,7^{\mathrm{b}}$ & $20,0^{b}$ & 47 \\
\hline & ind.. & 822 & & & & & & \\
\hline \multirow[t]{3}{*}{ S. mystus } & mâles & 79 & $-16,102$ & 1,096 & 0,87 & $14,7^{\mathrm{a}}$ & $18,0^{\mathrm{a}}$ & 86 \\
\hline & femelles & 64 & $-10,109$ & 0,744 & 0,74 & $13,6^{\mathbf{b}}$ & $16,0^{b}$ & 65 \\
\hline & ind.. & 227 & & & & & & \\
\hline \multirow[t]{3}{*}{ S. schall } & mâles & 1615 & $-5,301$ & 0,434 & 0,94 & $12,2^{\mathrm{a}}$ & $20,0^{a}$ & 38 \\
\hline & femelles & 1197 & $-3,882$ & 0,293 & 0,84 & $13,2^{\text {b }}$ & $24,0^{\mathbf{b}}$ & 51 \\
\hline & ind.. & 2041 & & & & & & \\
\hline \multirow{3}{*}{ S. nigrita } & mâles & 319 & $-5,702$ & 0,437 & 0,94 & $13,0^{\mathrm{a}}$ & $22,0^{\mathrm{a}}$ & 58 \\
\hline & femelles & 419 & $-4,624$ & 0,353 & 0,97 & $13,1^{\text {a }}$ & $24,0^{\mathbf{b}}$ & 47 \\
\hline & ind.. & 439 & & & & & & \\
\hline
\end{tabular}

$\overline{L_{50}}=$ longueur totale en $\mathrm{cm}$ à laquelle $50 \%$ des poissons sont matures, $L_{100}=$ longueur totale en cm à laquelle $100 \%$ des poissons sont matures, ind. = poissons juvéniles dont le sexe n'a pu être déterminé, Ltmax = longueur totale maximale capturée ; a et b sont les paramètres du modèle logistique. Pour une même espèce, les chiffres qui portent les mêmes lettres $n e$ sont pas significativement différents entre eux ( $p>0,05)$. 
Tableau 4 : Caractéristiques de capture des six espèces de poisson-chat dans le delta de l'Ouémé à Agonlin Lowé.

\begin{tabular}{lcccccc}
\hline Paramètres de capture & C. gariepinus & C. ebriensis & S. intermedius & S. mystus & S. schall & S. nigrita \\
\hline $\mathrm{Lc}_{50}$ calculé $(\mathrm{cm})$ & 1,8 & 4,82 & 5,5 & 5,49 & 2,43 & 3,16 \\
$\mathrm{Lc}_{75}$ calculé $(\mathrm{cm})$ & 11,47 & 9,04 & 7,01 & 6,69 & 3,76 & 4,98 \\
$\mathrm{Lc}_{50} / \mathrm{L} \infty$ & 0,03 & 0,14 & 0,22 & 0,25 & 0,08 & 0,10 \\
$\mathrm{Lc}_{100}$ calculé $(\mathrm{cm})$ & 23,6 & 18,2 & 9,7 & 8,0 & 5,0 & 19,4 \\
$\mathrm{Lt}$ calculée à 1 an & 23,6 & 10,5 & 8,6 & 15,6 & 12,2 & 13,5 \\
$\mathrm{Lt}$ max observée à $(\mathrm{cm})$ & 64,0 & 33,0 & 20,6 & 20,8 & 32,1 & 27,6 \\
$\mathrm{~L} \infty\left(\mathrm{Lt} \mathrm{en} \mathrm{cm}^{2}\right.$ & 66,08 & 34,31 & 24,68 & 21,53 & 32 & 32 \\
\hline $\mathrm{Lc}_{50}, \mathrm{Lc}_{75}$ et $\mathrm{Lc}_{100}=$ respectivement longueur totale $(\mathrm{cm})$ à laquelle 50\%, 75\% et 100\% des poissons sont capturés.
\end{tabular}

\section{DISCUSSION}

A l'exception de l'espèce $S$. mystus, la $\mathrm{L}_{50}$ chez les mâles est inférieure à la $\mathrm{L}_{50}$ chez les femelles. Ainsi, les mâles mâtures à une taille inférieure à celle des femelles.

Chez C. gariepinus, les tailles de première maturité observées $(16,2 \mathrm{~cm}$ pour les mâles et $18,1 \mathrm{~cm}$ pour les femelles) sont très différentes de celles trouvées ailleurs. Bruton et Allanson (1980) ont trouvé dans le Lac Sibaya (Afrique du Sud) une $\mathrm{L}_{50}$ de $28,0 \mathrm{~cm}$, tandis que dans les retenues d'eau du Burkina Faso, Baijot et al. (1994) indiquaient une $\mathrm{L}_{50}$ de $35,0 \mathrm{~cm}$ pour les mâles et de $37,5 \mathrm{~cm}$ pour les femelles. En Afrique du Sud, Potgieter (1974 cité par Chikou, 2006) et Van der Waal (1975) ont rapporté respectivement des $\mathrm{L}_{50}$ de $37 \mathrm{~cm}$ (mâle) et $38 \mathrm{~cm}$ (femelle) et 42,6 cm (mâle) et $41,4 \mathrm{~cm}$ (femelle). Au lac Liambezi (Namibie), Van der Waal (1974) a trouvé une $\mathrm{L}_{50}$ de $47 \mathrm{~cm}$ pour les mâles et de 49,6 cm pour les femelles, tandis que Willoughby et Tweddle (1978) ont rapporté dans la vallée de la rivière Shire (Malawi) une $\mathrm{L}_{50}$ de $32 \mathrm{~cm}$ pour les mâles et de $29 \mathrm{~cm}$ pour les femelles de la même espèce. Ces différences avec nos résultats seraient liées à la condition du poisson dans le milieu et à la forte pression de pêche qui s'exerce sur ses populations.

Chez C. ebriensis, les tailles de première maturité observées sont de $7,4 \mathrm{~cm}$ pour les mâles et de $11,3 \mathrm{~cm}$ pour les femelles. Dans l'ensemble, la $\mathrm{L}_{50}$ pour l'espèce C. ebriensis dans la vallée de l'Ouémé reste très faible en comparaison de celles trouvées en zone forestière dans la rivière Anambra par Ezenwaji (1992, 2002). En effet, cet auteur signale que plus de 50\% d'individus des deux sexes sont matures entre 20,1 et $25,0 \mathrm{~cm}$. Cette différence serait liée à la condition des poissons et donc aux caractéristiques des habitats, les sujets collectés en zone forestière au Nigeria ayant probablement une croissance plus rapide $\mathrm{du}$ fait de la disponibilité alimentaire toute l'année. On pourrait penser que dans les Whédos du delta de l'Ouémé, l'espèce présente, comme chez les Cichlidae en situation stressante de surpopulation, un phénomène de "nanisme" qui l'amène à se reproduire à une taille très faible, ce qui expliquerait assez bien les $\mathrm{L}_{50}$ trouvées. Les tailles de première maturité de plusieurs espèces africaines ont été étudiées par Paugy et Lévêque (1999), mais les résultats sont très variables selon les espèces et n'incluent pas $C$. ebriensis. La différence entre les $\mathrm{L}_{50}$ des deux sexes chez $C$. ebriensis est tellement importante qu'elle pourrait être due à un dimorphisme sexuel de la croissance et de la mortalité comme suggéré par Wootton en 1998.

Chez S. intermedius les $\mathrm{L}_{50}$ sont de 8,4 $\mathrm{cm}$ pour les mâles et de $9,7 \mathrm{~cm}$ pour les femelles. Elles sont inférieures à la $\mathrm{L}_{50}$ trouvée par Lalèyè et al. (2004) pour l'ensemble du bassin de l'Ouémé, soit 11,5 $\mathrm{cm}$ et $10,1 \mathrm{~cm}$ Lt respectivement pour les mâles et les femelles. Dans le delta de Okavango (Botswana), Merron et Mann (1995) ont trouvé $14,3 \mathrm{~cm}$ et $17,3 \mathrm{~cm} \mathrm{Ls}$ (longueur standard) respectivement pour les mâles et les femelles. La très faible $\mathrm{L}_{50}$ trouvée chez S. intermedius à Agonlin lowé serait liée aux conditions du milieu et indique sa forte exploitation dans la station. 

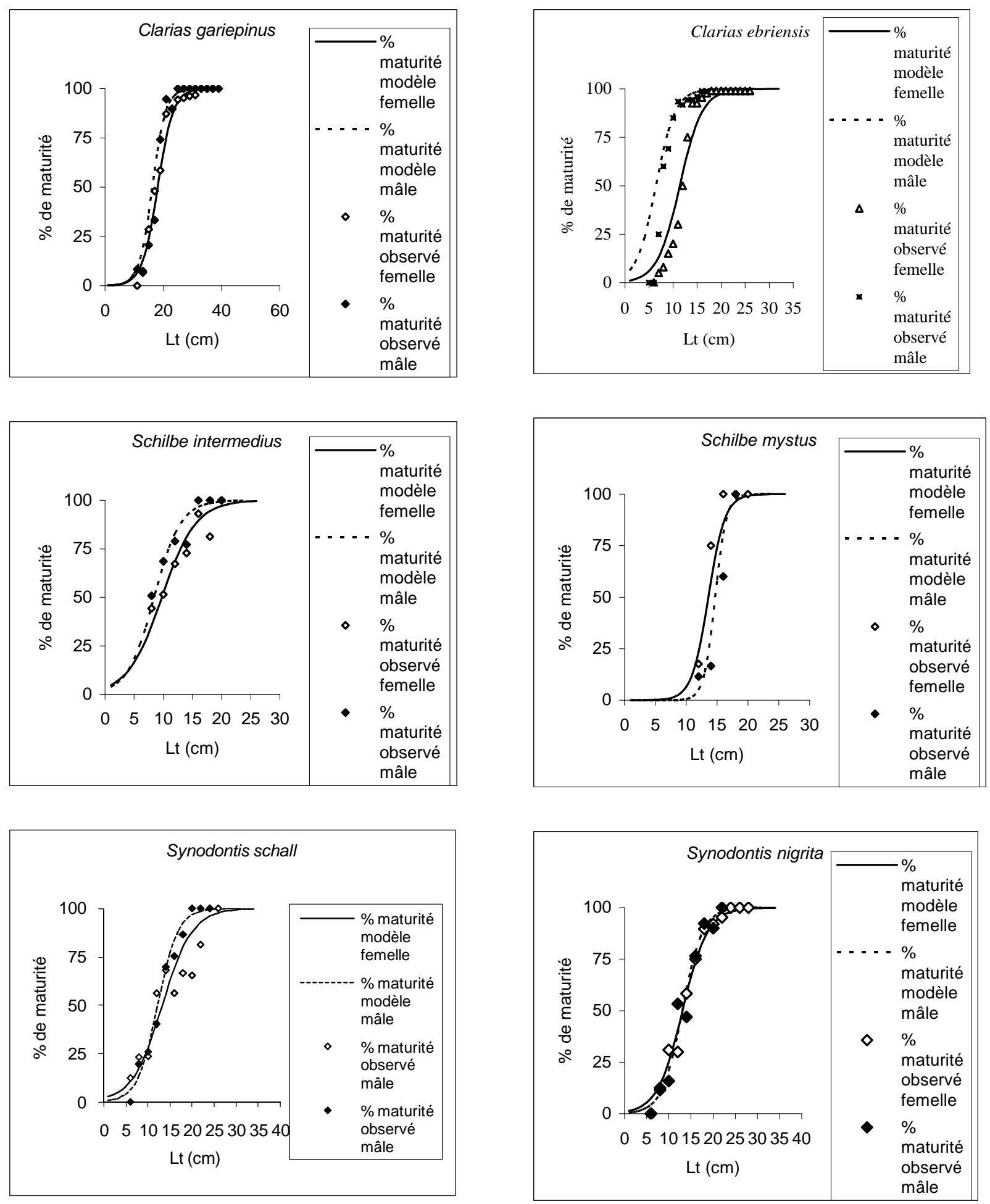

Figure 2 : Pourcentage de maturité sexuelle en fonction de la longueur totale du corps (Lt en $\mathrm{cm}$ ) chez les six espèces de poisson étudiées à Agonlin Lowé et courbe d'ajustement (modèle logistique). 
Chez $S$. mystus les $\mathrm{L}_{50}$ trouvées sont de $14,7 \mathrm{~cm}$ pour les mâles et de $13,6 \mathrm{~cm}$ pour les femelles. Elles sont plus élevées que celles trouvées dans la plupart les cours d'eau de la sous-région. En Côte d'Ivoire, Lévèque et Herbinet (1980) ont trouvé une $\mathrm{L}_{50}$ de 11,0 $\mathrm{cm}$, tandis que Albaret (1982) a trouvé une $\mathrm{L}_{50}$ de 10,0 cm. Dans les retenues d'eau du Burkina Faso, Baijot et al. (1994) ont trouvé $13,2 \mathrm{~cm}$ pour les mâles et $11,2 \mathrm{~cm}$ pour les femelles. Dans le lac Tchad, Mok (1975) a trouvé que la plus petite femelle en maturation avancée mesurait $12,0 \mathrm{~cm}$. Une autre femelle pré-pubère de $9,6 \mathrm{~cm}$ a été également observée par l'auteur, tandis que le plus petit mâle pubère est un individu de 11,5 cm. Dans l'Ogoun au Nigeria, Adebisi (1987) a observé une $\mathrm{L}_{50}$ de $24,6 \mathrm{~cm}$ qui est quand même supérieure à celle observée à Agonlin Lowé. De ces résultats, il semblerait que $S$. mystus présente une meilleure condition à Agonlin Lowé.

Chez $S$. schall et $S$. nigrita, les $\mathrm{L}_{50}$ trouvées sont respectivement de $12,2 \mathrm{~cm}$ et $13,0 \mathrm{~cm}$ pour les mâles et $13,2 \mathrm{~cm}$ et $13,1 \mathrm{~cm}$ pour les femelles. Ces valeurs sont largement inférieures à celles observées par Lalèyè et al. (2007), soit respectivement $15,0 \mathrm{~cm}$ et 21,0 $\mathrm{cm}$ pour les mâles et $16,0 \mathrm{~cm}$ et $22,0 \mathrm{~cm}$ pour les femelles pour tout le bassin de l'Ouémé. Cette différence pourrait provenir du fait que le travail des auteurs a porté sur tout le bassin de l'Ouémé et que les poissons du nord ont des conditions plus élevées (Lalèyè et al., 2007) et arriveraient à maturité plus tard que ceux du delta soumis à une forte pression de pêche. Ailleurs dans la sous-région pour $S$. schall, Albaret (1982) a trouvé 15,0 cm en Côte d'Ivoire, Adebisi (1987) 29,1 cm dans l'Ogoun au Nigeria et Ofori-Danson (1992) 20,0 cm dans la Volta.

En ce qui concerne les tailles de première capture $\left(\mathrm{Lc}_{50}\right)$, elles sont en dessous de la taille de première maturité pour les six populations étudiées. Les modes d'exploitation des poissons dans le delta de l'Ouémé tendent à réduire la fécondité des stocks car la plupart des poissons capturés n'ont pas la chance d'atteindre leur taille compatible avec une bonne reproduction. Il serait intéressant de capturer les poissons à une plus grande taille.

\section{Conclusion}

L'étude réalisée est tout à fait concluante et a permis de renseigner sur le statut des stocks pour les six espèces de poissons-chats étudiées. Elle pourrait être élargie à toutes les espèces exploitées dans le delta de l'Ouémé en vue d'une approche de gestion plus globale. A court terme, il serait intéressant, au plan de la gestion de la pêche, de faire respecter la réglementation en vigueur et de réduire les captures en début de crue lors des migrations de reproduction en vue de la protection des reproducteurs. L'amélioration de la connaissance de la reproduction artificielle des six espèces et le repeuplement du cours d'eau constituent des pistes à moyen et long termes.

\section{REMERCIEMENTS}

L'étude a été réalisée dans le cadre du programme CIUF «Initiatives propres, Université de Liège» "Biodiversité et Aquaculture des Poissons-chats du Bénin" financé de 1998 à 2003 par la Direction Générale de la Coopération Internationale (DGCI) de Belgique. Les auteurs sont reconnaissants à tous les chercheurs du Laboratoire d'Hydrobiologie et d'Aquaculture (LHA) de la Faculté des Sciences Agronomiques de l'Université d'AbomeyCalvi au Bénin pour leur assistance à la collecte des données ainsi qu'aux agents des pêches et aux pêcheurs du village d'Agonlin Lowé pour leur coopération.

\section{BIBLIOGRAPHIE}

Adam KS, Boko M. 1993. Le Bénin. Memb, Edicef : Paris.

Adebisi AA. 1987. The relationships between the fecundities, gonado-somatic indices and egg sizes of some fishes of the Ogun River, Nigeria. Hydrobiologia, 111: 151156.

Albaret J-J. 1982. Reproduction et fécondité des poissons d'eau douce de Côte 
d'Ivoire. Rev. Hydrobiol. Trop., 15(4): 347-371.

Baijot E, Moreau J, Barry I, Bouda S. 1994. Biologie et démographie des principales espèces de poissons des retenues d'eau du Burkina Faso. In Aspects Hydrobiologiques et Piscicoles des Retenues en Zone Soudano-Sahélienne, Baijot E, Moreau J, Bouda S (eds). CTA: Wageningen; 87-122.

Bruton MN, Allanson BR. 1980. The growth of Clarias gariepinus in Lake Sibaya, South Africa. S. Afr. J. Zool., 15: 7-15.

Chikou A. 2006. Etude de la démographie et de l'exploitation halieutique de six espèces de poissons-chats (Teleostei, Siluriformes) dans le delta de l'Ouémé au Bénin. Thèse de Doctorat, Université de Liège, Liège, p. 459.

Chikou A, Lalèyè $\mathrm{P}$, Philippart J-C, Vandewalle P. 2007. Quelques aspects de la biologie de reproduction chez Clarias ebriensis Pellegrin (Siluriformes, Clariidae) dans le delta de l'Ouémé au Bénin (Afrique de l'Ouest). Cybium, 31(2): 207-215.

Chikou A, Lalèyè $\mathrm{P}$, Raemakers V, Vandewalle P, Philippart J-C. 2008. Etude de l'âge et de la croissance chez Clarias gariepinus (Pisces, Clariidae) dans le delta de l'Ouémé au Bénin (Afrique de l'Ouest). Int. J. Biol. Chem. Sci., 2(2): 157-167.

Dagnelie P. 1973. Théorie et Méthodes Statistiques : Applications Agronomiques (Vol. 1). Presses Agronomiques de Gembloux : Belgique.

Dunn IG. 1972. The commercial fishery of Lake George, Uganda (East Africa). Afr. J. Trop. Hydrobiol. \& Fish., 2(2): 109120.

Ezenwaji HMG. 1992. The reproductive biology of four African catfishes (Osteichthyes: Clariidae) in Anambra River basin, Nigeria. Hydrobiologia, 242: 155-164.

Ezenwaji HMG. 2002. The biology of Clarias ebriensis Pellegrin, 1920 (Osteichthyes :
Clariidae) in an Africa rainforest river basin. Fish. Res., 54: 235-252.

Gwahaba JJ. 1978. The biology of cichlid fishes (Teleostei) in an equatorial lake (Lake George, Uganda). Arch. Hydrobiol., 83(4): 538-551.

Imorou TI, Fiogbe ED, Koukpodé B, Kestemont P. 2007. Rearing of African catfish (Clarias gariepinus) and vundu catfish (Heterobranchus longifilis) in traditional fish ponds (whedos) : Effect of stocking density on growth, production and body composition. Aquaculture, 262: 65-72.

Imorou TI. 2007. Amélioration de la production halieutique des trous traditionnels à poissons (whédos) du delta de l'Ouémé (sud Bénin) par la promotion de l'élevage des poissons-chats Clarias gariepinus et Heterobranchus longifilis. Thèse de Doctorat, Université de Namur, Belgique, p. 186.

Lalèyè P. 1995. Ecologie comparée de deux espèces de Chrysichthys, poissons Siluriformes (Claroteidae) du complexe lagunaire «Lac Nokoué-Lagune de Porto-Novo » au Bénin. Thèse de Doctorat, Université de Liège, Liège, p. 199.

Lalèyè P, Philippart J-C, Heymans J-C. 1995. Cycle annuel de l'indice gonadosomatique et de la condition chez deux espèces de Chrysichthys (Siluriformes, Bagridae) au lac Nokoué et à la lagune de Porto-Novo au Bénin. Cybium, 19(2): 131-142.

Lalèyè $\mathrm{P}$, Chikou A, Philippart J-C, Teugels GG, Vandewalle P. 2004. Etude de la diversité ichtyologique du bassin du fleuve Ouémé au Bénin (Afrique de l'Ouest). Cybium, 28(4): 329-339.

Lalèyè P, Akélé D, Philippart J-C. 2007. La pêche traditionnelle dans les plaines inondables du fleuve Ouémé au Bénin. Cahiers d'Ethologie, 22(2): 25-38.

Lalèyè $\mathrm{P}$, Salako $\mathrm{O}$, Chikou A, Philippart J-C. 2005. Artisanal gill-net fishery catches of the catfish, Schilbe intermedius (Teleostei: Schilbeidae), in two tributaries 
of the Ouémé River, Bénin, West Africa. African Journal of Aquatic Science. 30(2): 163-166.

Lalèyè $\mathrm{P}$, Chikou $\mathrm{A}$, Gnohossou $\mathrm{P}$, Vandewalle $\mathrm{P}$, Philippart J-C, Teugels GG. 2006. Studies on the biology of two species of catfish Synodontis schall and Synodontis nigrita (Ostariophysi : Mochokidae) from the Ouémé River, Bénin. Belgian Journal of Zoology, 136(2): 193-201.

Lévêque C, Herbinet P. 1980. Caractères méristiques et biologie de Schilbe mystus (Pisces, Schilbeidae) en Côte d'Ivoire. Cah. Orstom sér. Hydrobiol., 13(3-4): 161-170.

Mok M. 1975. Biométrie et biologie des Schilbe (Pisces, Siluriformes) du bassin tchadien. II ${ }^{\mathrm{ème}}$ partie. Biologie comparée des deux espèces. Cah. Orstom Sér. Hydrobiol., 9(1): 33-60.

Merron GS, Mann BQ. 1995. The reproductive and feeding biology of Schilbe intermedius Rüppell in the Okavango Delta, Botswana. Hydrobiologia, 308: 121-129.

Micha J-C. 1973. Etude des populations piscicoles de Oubangui et tentatives de sélection et d'adaptation de quelques espèces à l'étang de pisciculture. Thèse de Doctorat, Université de Liège, Liège, p. 110.

Moreau J. 1979. Biologie et évolution des peuplements de cichlides (pisces) introduits dans les lacs malgaches d'altitude. Thèse de Doctorat, Institut National Polytechnique de Toulouse, p. 301.

Niyonkuru C. 2007. Etude comparée de l'exploitation et de la démographie des poissons Cichlidés dans les lacs Nokoué et Ahémé au Bénin. Thèse de Doctorat, Université d'Abomey-Calavi, Bénin, p. 199.

Ofori-Danson PK. 1992. Ecology of some species of catfish Synodontis (Pisces : Mochokidae) in the Kpong Headpond in Ghana. Environmental Biology of Fishes, 35: 49-61.

Paugy D, Lévêque. C. 1999. La reproduction. In Les Poissons des Eaux Continentales Africaines: Diversité, Ecologie, Utilisation par l'Homme, Lévêque C, Paugy D (eds). Editions IRD: Paris; 129151.

Tweddle D, Turner JL. 1977. Age, growth and natural mortality rates of some cichild fishes of Lake Malawi. J. Fish Biol., 10: 385-398.

Van der Waal BCW. 1974. Observations on the breeding habits of Clarias gariepinus (Burchell). J. Fish Biol., 6: 23-27.

Van der Waal BCW, Schoonbee HJ. 1975. Age and growth of Clarias gariepinus (Burchell) in the Transvaal. South Africa Journal of Fish Biology, 7: 227-233.

Von Bertalanffy L. 1938. A quantitative theory of organic growth (inquiries on growth laws II.). Hum. Biol., 10(2): 181213.

Welcomme RL. 2002. An evaluation of tropical brush and vegetation park fisheries. Fish. Manage. Ecol., 9(3): 175188.

Willoughby NG, Tweddle D. 1978. The ecology of the catfish Clarias gariepinus and Clarias ngamensis in the Sbire Valley, Malawi. Journal of Zoology, 186: 507-534.

Wootton RJ. 1998. Ecology of Teleost Fishes $\left(2^{\text {nd }}\right.$ edn). Kluwer Academic Publishers: London. 